\title{
Photocurrent in conjugated polymers
}

\author{
Baruch Horovitz and Ehoud Pazy \\ Department of Physics and Ilze Katz center for nanotechnology, Ben-Gurion University of the Negev, Beer-Sheva 84105, Israel
}

\begin{abstract}
Nonlinear photocurrent carriers in conjugated polymers, such as polarons, bipolarons and solitons, are considered at low photon energies where a tunnelling process is necessary. We show that polarons usually dominate the photocurrent $I$ due to a novel electric field assisted tunnelling for which $\ln I \sim-E^{-2 / 3}$. For near degenerate polymers an electric field $E$ which exceeds the confinement potential and frequencies above twice the soliton energy, soliton tunnelling is favored. Photocurrent data can then be used to identify the remarkable phenomenon of soliton conduction.
\end{abstract}

Photoexcitation of charge carriers in conjugated polymers is of considerable interest for determining types of charge carriers and for probing novel conduction mechanisms. Considerable theoretical attention has been given to polyacetylene (PA) [1-4] which has degenerate ground states (i.e. by inverting the dimerization sign) predicting photocurrent carried by charged solitons. Experimentally, however, significant photocurrent has been observed in non-degenerate conjugated polymers such as polydiacetylene (PDA) $[5,6]$.

In a non-degenerate polymer the dimerization pattern in the ground state is unique, being fixed by the polymer structure. The charge carriers are expected to be [4] either polarons or doubly charged bipolarons; the continuum model for polarons and bipolarons has been considered in detail [7-9]. Reversing the sign of the dimerization leads to a local minimum (at least for near degenerate systems) which is higher by an energy of $\alpha$ per unit length. A soliton is an excitation which interpolates between states with opposite signs of dimerization; hence generating a soliton pair leads to an excess energy linear in the separation $R$ of the solitons, i.e. a confinement potential $\alpha R$. In contrast, polarons or bipolarons are local deformations of the ground state, hence a polaron pair has no confinement potential. We note that interchain coupling acts as a confinement potential, hence even PA with degenerate minima should be considered as a neardegenerate polymer.

Some properties of PDA serve us as a prime example for illustrating phenomena in a non-degenerate polymer. PDA has chains of the form $[=R C-C \equiv C-C R=]_{n}$ where $\mathrm{R}$ is one of a number of side groups with which the monomer can be synthesized. Band structure calculations [10] show that the states near the gap are essentially $\pi_{z}$ orbitals. If these orbitals were removed the underlying structure would be $[-R C-C=C-C R-]_{n}$, i.e. 3 bonds per carbon. If $a$ is the mean spacing of carbons, the electron spectrum in an extended zone scheme has gaps at $\pm n \pi / 4 a$ with $n=1,2,3$ since the unit cell has 4 carbons. Filling in one electron per Carbon for the $\pi_{z}$ orbitals up to wavevectors $\pm \pi / 2 a$ leads therefore to a gap at the Fermi level, a so called "extrinsic gap" $\Delta_{e}$. In addition, the $\pi_{z}$ orbitals tend to dimerize, i.e. their overlap between nearest carbons is alternating. This dimerization increases the gap at the Fermi level so that the gain in electron energies overcomes the cost in the lattice distortion. For PDA, the usual acetylenic ground state is then formed with the above triple bond. It is possible, however, depending on the side groups $\mathrm{R}$, on temperature or on external strain, that the ground state will favor the opposite sign for this dimerization, leading to the butatrienic form $[-R C=C=C=C R-]_{n}$. In fact, some data supports a PDA butatrienic form produced in a different photopolymerization procedure, which transforms into an acetylenic form when annealed above $\approx 350 \mathrm{~K}$ [11]. Solitons in PDA interpolate between these states, i.e. $[\ldots-R C=C=C=C R-R \dot{C}-C \equiv C C R=\ldots]$. The central carbon $\dot{C}$ has only three bonds, i.e. it acquires a localized orbital with intragap energy. If a phase transition in PDA is indeed posible, then at the critical temperature $\alpha=0$ and solitons can lead to photocurrent [12]. As we show below, even a small $\alpha$ can demonstrate the remarkable phenomenon of soliton conductance.

Experimental data on PDA [5] with currents measured down to $10^{-16} A$ shows a steep photocurrent edge at photon energy of $0.8 \mathrm{eV}$ which is well below either the band gap of $2.4 \mathrm{eV}$, or the exciton level at $2 \mathrm{eV}$. Further data on PDA with a different side group [6] has shown that thermal annealing allows for measurable photocurrents at $2.2 \mathrm{eV}$, with an exciton level at $2.3 \mathrm{eV}$; the current sensitivity in this case was about $10^{-13} \mathrm{~A}$.

In the present work we study photocurrent due to tunnelling into a variety of charge carriers - polarons, bipolarons and solitons. We show that at low photon energies $\hbar \omega<2 E_{p}$ polarons usually dominate, where $E_{p}$ is the polaron formation energy. The electric field allows for a novel tunnelling route where weak lattice deformations are formed at large separation $R_{p}$ such that upon charge transfer shallow polarons are formed; the energy gain $e E R_{p}$ compensates for the missing formation energy $2 E_{p}-\hbar \omega$. This process is likely to be effective also in semiconductors with higher dimensionality. We also show that bipolarons can be directly photoproduced, though with a smaller probability. Finally we show that 
the external electric field $E$ can overcome the confinement potential when $e E>\alpha$ and allows for charged soliton creation. We find that for $2 E_{s}<\hbar \omega<2 E_{p}$, and above the threshold field $\alpha / e$ soliton formation is favored, where $2 E_{s}$ is the soliton pair energy (in addition to the $\alpha R$ term). This process can serve as a clear identification of soliton conduction, adding to intriguing PA data [4].

We consider first photocurrent due to polarons at $\hbar \omega<2 E_{p}$. The tunnelling process is parameterized by two local deformations of the dimerization pattern separated by distance $R$, each deformation yields a bound state of size $1 / \kappa$. These localized states are pulled from the valence and conduction band edges at $\pm \Delta_{0}$, respectively. We assume that direct electron-electron (e-e) interactions are embedded in the parameters such as $\Delta_{0}$, $E_{p}$ and $\kappa$; where needed we address e-e interactions explicitly. For the purpose of illustration, we show the electron level structure in Fig. 1 for the electron-lattice (e-l) model in the absence of e-e interactions.

The initial state potential $V_{0}(\kappa, R)$ describes the deformed lattice with the electron occupation following adiabatically that of the ground state. The excited state corresponds to a dipole allowed transition of one electron into a state with energy higher by $2 \omega_{0}$, e.g. for $\kappa=0$ we have $2 \omega_{0}=2 \Delta_{0}$. The excited state can relax adiabatically into a two polaron state $P^{+} P^{-}$with energy minimized at some $\kappa=\kappa_{p}$, i.e. the polaron energy $E_{p}(\kappa)$ relaxes to $E_{p} \equiv E_{p}\left(\kappa_{p}\right)$. In the e-l model the initial state of each deformation has the lower level (Fig. 1a) doubly occupied while the upper level is empty; the excited state has one more electron in the upper state $\left(P^{-}\right)$or one less in the lower state $\left(P^{+}\right)$.

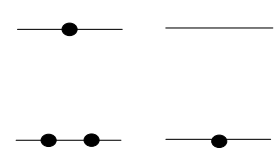

(a)

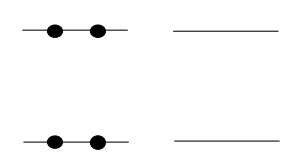

(b)
FIG. 1. Two localized states with intragap energies $\pm \omega_{0}$ (continuum states starting at $\pm \Delta_{0}, \Delta_{0}>\omega_{0}$, are not shown). The dots represent occupation of these levels in the e-l model for (a) a separated polaron pair $P^{-} P^{+}$, (b) a separated bipolaron pair $B^{+} B^{-}$.

The condition for having a localized state with well defined charge is (i) $R \gg 1 / \kappa$ and (ii) that $E$ does not mix the state with continuum states, i.e. the energy shift of the localized state $\approx e E / \kappa$ is small relative to the excitation energy into the continuum, i.e. $e E / \kappa \ll \Delta_{0}-\omega_{0}$. We claim that for small $\kappa$ the latter is $\Delta_{0}-\omega_{0} \approx \Delta_{0} \kappa^{2} \xi^{2}$ where $\xi=v_{F} / \Delta_{0}$ and $v_{F}$ is the Fermi velocity in the absence of an energy gap. For the noninteracting case this is seen by continuing analytically the momentum $k \rightarrow i \kappa$ in the spectrum $\omega_{0}=\sqrt{\Delta_{0}^{2}-v_{F}^{2} \kappa^{2}} \approx \Delta_{0}\left(1-\frac{1}{2} \kappa^{2} \xi^{2}\right)$. In the final paragraph before the conclusions we show that to first order in Coulomb interactions the $\kappa^{2}$ form is maintained. This is rather surprising since the Hartree term by itself gives $\mathrm{a} \sim \kappa$ term [4]. The perturbation parameter being $e^{2} \kappa / \Delta_{0}$ implies that higher order terms lead to higher powers of $\kappa$.

The condition $e E / \kappa \ll \Delta_{0}-\omega_{0}$ becomes then $\kappa \gg \kappa_{m}$ where

$$
\kappa_{m}=\left(e E \xi / \Delta_{0}\right)^{1 / 3} / \xi
$$

Since typically $\Delta_{0} \approx 1 \mathrm{eV}, \xi \approx 1 \mathrm{~nm}$ and $E \lesssim 10^{5} \mathrm{~V} / \mathrm{cm}$ we have $e E \xi / \Delta_{0} \lesssim 10^{-2}$ which serves as a small parameter. This condition for localized charges is extremely important - it allows the excited state potential to gain an electric field energy $V_{e x}(\kappa, R)=2 E_{p}(\kappa)-e E R$, hence the energy gain $e E R$ facilitates tunnelling even if $\hbar \omega<2 E_{p}$. The initial state potential has neutral components, hence $V_{0}(\kappa, R)=2 E_{p}(\kappa)-2 \omega_{0}(\kappa)$.

Perturbation theory in the exciting photons $\sim e^{i \omega t}$ $[3,13]$ in the adiabatic limit $\left(\hbar \omega, 2 \Delta_{0}\right.$ large compared with a typical phonon energy $\hbar \omega_{p h}$ ) shows that the electron transition occurs at $\kappa, R$ such that $V_{0}(\kappa, R)=$ $V_{e x}(\kappa, R)-\hbar \omega$. The tunnelling barrier changes then from $V_{0}(\kappa, R)$ to $V_{e x}(\kappa, R)-\hbar \omega$ at the crossing point. The dynamics is dominated by the ion kinetic energy; the space dependent dimerization pattern $\Delta(x ; k, R)=$ $\Delta_{p}(\kappa, x-R / 2)+\Delta_{p}(\kappa, x+R / 2)$, where $\Delta_{p}(\kappa, x)$ is the single polaron pattern, leads to the kinetic energy

$$
\int d x \frac{\dot{\Delta}^{2}}{\pi \lambda v_{F} \omega_{p h}^{2}}=M_{1}(\kappa) \dot{R}^{2} / 2+M_{2}(\kappa) \dot{\kappa}^{2} /\left(2 \kappa^{4}\right)
$$

where dot is a time derivative and $\lambda$ is the electronphonon coupling. $M_{1}(\kappa)$ is the mass for polaron motion while $M_{2}(\kappa)$ is the mass for the polaron size $1 / \kappa$ modulations. Note that $\Delta_{p}(\kappa, x)$ is symmetric in $x$ hence $\partial_{R}$ terms are antisymmetric and the coefficient of a cross term $\dot{R} \dot{\kappa}$ vanishes.

Consider first a tunnelling trajectory such that initially $\kappa$ increases (at small $R<\xi$ ) until crossing into the excited potential occurs. The matrix element for the transition involves the photon field and a wavefunction overlap which is large for $R<\xi$. The tunnelling continues then on the excited state with increasing $R$, reducing the excited state energy with the $-e E R$ term until it vanishes at $R_{1}=\left(2 E_{p}-\hbar \omega\right) / e E$ (Fig. 2a). The condition $R_{1} \gg \xi$ is satisfied if $2 E_{p}-\hbar \omega$ is not too small, e.g. $\gtrsim 0.1 \mathrm{eV}$ and with $\xi \approx 1 \mathrm{~nm}$ one needs $E \ll 10^{6} \mathrm{~V} / \mathrm{cm}$. Most of the trajectory is then in the regime $R \gg \xi$ justifying the use of the form $V_{e x}(\kappa, R)=2 E_{p}-e E R$ and the use of effective masses. The tunnelling rate along the $R$ trajectory under the barrier $2 E_{p}-e E R-\hbar \omega$ (curve p in Fig. 2a) is given by a WKB form $\exp \left\{-2 \int_{0}^{R_{p}}\left[M_{p}\left(2 E_{p}-e E R-\hbar \omega\right)\right]^{1 / 2} d R\right\}$ where $M_{p}=M_{1}\left(\kappa_{p}\right)$ is the polaron mass; hence

$$
\Gamma_{1} \sim e^{-\frac{4 \sqrt{M_{p}}}{3 e E}}\left(2 E_{p}-\hbar \omega\right)^{3 / 2} .
$$


This exponent involves two large parameters; first an electric field term, $\Delta_{0} / e E \xi>10^{2}$, and second an adiabatic parameter $\sqrt{M_{p} v_{F}^{2} / \Delta_{0}} \sim \Delta_{0} / \omega_{p h} \approx 10$. Hence $\Gamma_{1}$ is extremely small.

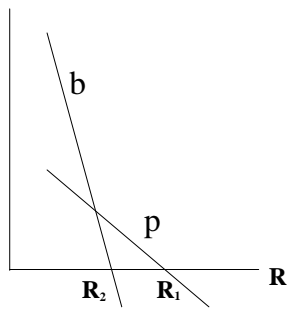

(a)

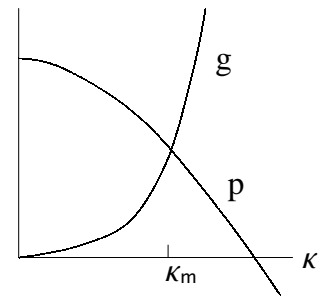

(b)
FIG. 2. Energy barriers for (a) tunnelling along $R$ for polarons (curve $p$ with $2 E_{p}-\hbar \omega-e E R$ ) and for bipolarons (curve $b$ with $2 E_{b}-\hbar \omega-2 e E R$ ), and (b) tunnelling along $\kappa$ : the initial deformed ground state energy (curve $g$ with $\left.2 E_{p}(\kappa)-2 \omega_{0}(\kappa)\right)$ crosses into the polaron state (curve $p$ with $\left.2 E_{p}(\kappa)-e E R-\hbar \omega\right)$ at the minimal $\kappa$ value of $\kappa_{m}$.

To find the optimal tunnelling trajectory in the $R, \kappa$ plane and avoid the strong suppression of tunnelling along $R$ (Eq. 3) we consider the opposite extreme where $\kappa$ increases from 0 at a large fixed $R$. This corresponds to nucleating two lattice deformations at a large distance $R$ with initial energy $2 E_{p}(\kappa)-2 \omega_{0}(\kappa)$ which crosses the excited state energy at some $\kappa$ (Fig. $2 \mathrm{~b}$ ). The price to pay in this process is that the matrix elements involve the overlap of localized wavefunctions separated by a large $R$, i.e. $\ln \Gamma \approx-2 \kappa R$. As discussed above, the minimal $\kappa$ that is consistent with localized charges is $\kappa_{m}$, hence we choose to nucleate the deformations at a distance $R_{p}$ such that the crossing in Fig. (2b) is at $\kappa_{m}$, i.e.

$$
2 E_{p}\left(\kappa_{m}\right)-2 \omega_{0}\left(\kappa_{m}\right)=2 E_{p}\left(\kappa_{m}\right)-\hbar \omega-e E R_{p} .
$$

Since $\kappa_{m}$ is small $R_{p} \approx\left(2 \Delta_{0}-\hbar \omega\right) / e E$, hence the tunnelling rate is

$$
\Gamma_{p} \sim e^{-4\left(1-\frac{\hbar \omega}{2 \Delta_{0}}\right)\left(\frac{\Delta_{0}}{e E \xi}\right)^{2 / 3}}
$$

$\Gamma_{p}$ involves also the tunnelling across small $\kappa$ values of Fig. 2b which contributes a small correction within the exponent in Eq. (5). We note that if Coulomb energies would give an excitation energy $\sim \kappa$ (e.g. in semiconductors with higher dimensionality) then $\kappa_{m} \sim E^{1 / 2}$ and $\ln I \sim\left(\Delta_{0} / e E \xi\right)^{1 / 2}$. We also note that choosing $R<R_{p}$ for Fig. 2 implies a higher $\kappa$ so that the above $\kappa_{m} R_{p}$ is the minimal value.

The result for $\Gamma_{p}$ is remarkable - it reduces the large $\Delta_{0} / e E \xi$ factor in Eq. (3) to its $2 / 3$ power and also avoids the large adiabatic parameter. Furthermore, this process is much more efficient than the Franz-Keldysh process [14] which is the photon assisted $\kappa=0$ process, i.e. tunnelling between extended states of the valence and conduction bands with $\ln \Gamma \sim-\left(1-\hbar \omega / 2 \Delta_{0}\right)^{3 / 2} \Delta_{0} / e E \xi$.
We conclude that the process leading to Eq. (5) is the most efficient one for generating polarons. This is a most remarkable process - the system prepares localized states at a large separation (typically $R_{p}>100 \mathrm{~nm}$ ) so as to accommodate an eventual transfer of charge.

We consider next tunnelling into bipolarons. The electric field breaks inversion symmetry and allows charge transfer between polarons, leading to doubly charged bipolarons. The excitaion potential is $V_{b}(\kappa, R)=$ $2 E_{b}(\kappa)-2 e E R$ where $2 E_{b}$ is the creation energy of two bipolarons; in the e-l model $2 E_{b}(\kappa)=2 E_{p}(\kappa)+2 \omega_{0}$ (Fig. $1 b)$. If tunnelling along $\mathrm{R}$ had dominated, then direct tunnelling into bipolarons would have dominated at low frequencies. To see this, note that $V_{b}(\kappa, R)-\hbar \omega$ crosses 0 at $R_{2}=\left(2 E_{b}-\hbar \omega\right) / 2\left(E_{b}\right.$ here is the minimized $\left.E_{b}(\kappa)\right)$ which is smaller than $R_{1}$ (Fig. 2a) if $\hbar \omega<4 E_{b}-2 E_{p}$, or $\hbar \omega \lesssim 0.4 \Delta_{0}$ in the e-l model. Tunnelling directly into bipolarons eliminates the more costly tunnelling range between $R_{2}$ and $R_{1}$ at the expense of an $e^{-2 \kappa R_{2}}$ factor for the second charge transfer. Hence, bipolarons would dominate at low $\hbar \omega$.

Within the much more efficient process of tunnelling along $\kappa$ at a large $R$ bipolarons are more efficiently generated when $R=R_{b}$ is chosen such that the bipolaron curve $2 E_{b}(\kappa)-2 e E R-\hbar \omega$ crosses the initial energy $2 E_{p}(\kappa)-2 \omega_{0}(\kappa)$ at the minimal $\kappa_{m}$, hence $R_{b} \approx$ $\left(2 \Delta_{0}-\frac{1}{2} \hbar \omega\right) / e E$. The polaron excitation energy is then below the bipolaron one, but since $-e E R$ is inefficient at $\kappa<\kappa_{m}$ the polaron curve would also cross near $\kappa_{m}$. The transfer of two charges involves now a $e^{-4 \kappa R}$ factor, hence $\Gamma_{b} \ll \Gamma_{p}$ with

$$
\Gamma_{b} \sim e^{-8\left(1-\frac{\hbar \omega}{4 \Delta_{0}}\right)\left(\frac{\Delta_{0}}{e E \xi}\right)^{2 / 3}}
$$

We consider next photocurrent of charged solitons which has been studied in detail for the degenerate PA case [1-4]. In the non-degenerate case, or for PA with interchain coupling, the electric field must exceed a threshold value to overcome the confinement potential. Solitons being topological objects must be generated by $R$ tunnelling - there is no $\kappa$ parameter or a local deformation which can generate a single soliton from the ground state. The excitation energy of a soliton pair $S^{+} S^{-}$is then $V_{s}(R)=2 E_{s}+\alpha R-e E R$ allowing tunnelling only for $e E>\alpha$. The tunnelling terminates at $R_{s}=\left(2 E_{s}-\hbar \omega\right) /(e E-\alpha)$ which is assumed to be large, $R_{s} \gg \xi$. The tunnelling rate into solitons with mass $M_{s}$, for $\hbar \omega<2 E_{s}, e E>\alpha$, is then

$$
\Gamma_{s} \sim \exp \left[-\frac{\sqrt{8 M_{s}}}{3(e E-\alpha)}\left(2 E_{s}-\hbar \omega\right)^{3 / 2}\right]
$$

The formation of $S^{+} S^{-}$leaves a metastable state which can then spontaneously form a pair of solitons without a photon. $\Gamma_{s}$ is extremely small (comparable to 
Eq. 3) unless $\hbar \omega$ is close to $2 E_{s}$. Soliton photocurrent may then dominate the polaron one only if the range $E_{s}<\hbar \omega<E_{p}$ exists. In the e-l model with weak $\alpha$ one has $E_{s} \approx 0.6 \Delta_{0}<E_{p} \approx 0.9 \Delta_{0}$ ( $E_{s}$ is defined so that a soliton pair energy separated by $R \gg \xi$ is $2 E_{s}+\alpha R$ ).

We consider therefore the range $2 E_{s}<\hbar \omega<2 E_{p}$. The polaron photocurrent is still given by Eq. (5) while soliton tunnelling can occur at $R<\xi$ where $\alpha$ and $E$ are ineffective. The result is then just as in the $\alpha=0$ system [3] $\ln \Gamma \approx-\Delta_{0} / \omega_{0}$, with weak $E$ dependence. We conclude that soliton photocurrent dominates over that of polarons when $2 E_{s}<\hbar \omega<2 E_{p}$ and $E>\alpha / e$. This is achievable if $\alpha / e \lesssim 10^{6} \mathrm{~V} / \mathrm{cm}$ or $\alpha=4 \Delta_{e} \Delta_{0} / \pi \lambda v_{F}$ [9] yields $\gamma \equiv \Delta_{e} / \lambda \Delta_{0} \lesssim 0.1$. Hence varying $E$ changes Eq. (5) at $E>\alpha / e$ into a larger and weakly $E$ dependent tunneling rate, exhibiting a sensitive tool for identifying the intriguing soliton conduction.

Finally, we present a somewhat technical section, evaluating the effect of Coulomb interactions to first order on the excitation energy of a polaron, e.g. $P^{+}$, leading to the important criteria for $\kappa_{m}$ in Eq. (1). The e-l system with (non-uniform) electron transfer between nearest neighbors has particle-hole symmetry, i.e. each eigenstate $\phi(n)$ at site $n$ with energy $E$ allows an eigenstate $(-)^{n} \phi(n)$ with energy $-E$. Completeness relation for the polaron $P^{+}$state (right side of Fig. 1a) yields for the charge density of each spin $\rho_{\uparrow}(n)=\frac{1}{2}$, $\rho_{\downarrow}(n)=\frac{1}{2}-\phi_{b}^{2}(n)$ where $\phi_{b}(n)$ is the lower energy localized eigenstate (Fig. 1). A Hubbard interaction, to first order is $\sum_{n}\left(\rho_{\uparrow}(n)-\frac{1}{2}\right)\left(\rho_{\downarrow}(n)-\frac{1}{2}\right)=0$; similarly all interactions with range of even sites vanish.

Consider next nearest neighbor interaction $\mathcal{H}_{V}$ with coupling $V$. Its average yields direct and exchange terms,

$$
\begin{aligned}
\left\langle\mathcal{H}_{V}\right\rangle= & \frac{1}{2} V \sum_{n}\left\{\left(\rho(n)-\frac{1}{2}\right)\left(\rho(n+1)-\frac{1}{2}\right)\right. \\
& \left.-\left|S+\phi_{b}^{*}(n) \phi_{b}(n+1)\right|^{2}-|S|^{2}\right\}
\end{aligned}
$$

where $\rho(n)=\rho_{\uparrow}(n)+\rho_{\downarrow}(n)$ and $S=\sum_{\alpha} \phi_{\alpha}^{*}(n) \phi_{\alpha}(n+1)$ sums on all occupied continuum states (per spin) with energy up to $-\Delta_{0}$. The 2 nd term above is the exchange for $\uparrow$ spin while the 3 rd is for $\downarrow$ spin. The summation can be done in a continuum limit [9] where $x=n a$ and $f_{\alpha}, g_{\alpha}$ are wavefunctions on the even and odd sites, respectively. For small $\kappa(1 / \kappa$ is the range of the localized state) we obtain from $[9]$

$$
S=\frac{\Delta_{e}-\Delta_{0}}{2 \pi \lambda v_{F}}-\left(1-\frac{1}{2} \gamma \xi \kappa\right) f_{b}(x) g_{b}(x)+O\left(\kappa^{2}\right)
$$

and the contribution to the polaron energy becomes

$$
\left\langle\mathcal{H}_{V}\right\rangle_{p}=V_{1}+V a \frac{\Delta_{e}-\Delta_{0}}{2 \pi \lambda v_{F}}\left(1-\frac{4}{\pi} \gamma \xi \kappa\right)+O\left(\kappa^{2}\right)
$$

where $V_{1} / N=-V a^{2}\left[\left(\Delta_{e}-\Delta_{0}\right) / 2 \pi \lambda v_{F}\right]^{2}$ is the correction to the ground state energy per site. Note that the direct term $\frac{1}{2} V \sum_{n} f_{b}^{2}(n) g_{b}^{2}(n) \sim O(\kappa)$ cancels with with the exchange term [Ref. [4] presents just the direct term which, as shown here, is insufficient].

The polaron $P^{+}$excited state involves an electron transfer from the top of the valence band with an extended wavefunction $\phi_{v}(n)$ into the lower localized state (Fig. 1a) which becomes doubly occupied. The direct term becomes $\frac{1}{2} V \sum_{n} \phi_{v}^{2}(n) \phi_{v}^{2}(n+1) \rightarrow 0$ for an extended system, the $\uparrow$ exchange is the same as in Eq. (8) while the $\downarrow$ exchange becomes $\left|S+\phi_{b}^{*}(n) \phi_{b}(n+1)-\phi_{v}^{*}(n) \phi_{v}(n+1)\right|^{2}$. Using [9] $\int d x f_{v}^{*}(x) g_{v}(x)=\frac{1}{2}+O\left(\kappa^{2}\right)$ we obtain that the excited polaron energy is the same as that of the polaron to order $\kappa^{2}$. We have also checked this conclusion within the continuum model with e-e interactions. Hence the excitation energy is $O\left(\kappa^{2}\right)$, the same as in the e-l model.

In conclusion, we have shown that polarons usually dominate the photocurrent when $\hbar \omega<2 E_{p}$ with the tunnelling rate from Eq. (5), i.e. $\ln I \sim-E^{-2 / 3}$. However, for $2 E_{s}<\hbar \omega<2 E_{p}$ and $E>\alpha / e$ solitons dominate the photocurrent. This is achievable for near-degenerate polymers, i.e. a small $\alpha$. Furthermore, the intriguing possibility that PDA has a phase transition at which the confinement parameter $\alpha$ vanishes [11] can be sensitively tested by the field dependence of photocurrent data.

Acknowledgements: We thank Doron Cohen and Amir Berman for stimulating discussions. This research was supported by THE ISRAEL SCIENCE FOUNDATION founded by the Israel Academy of Sciences and Humanities and by a German-Israeli DIP project.

[1] W. P. Su and J. R. Schrieffer, Proc. Natl. Acad. Sci. 77, 5626 (1980).

[2] R. Ball, W. P. Su and J. R. Schrieffer, J. Phys. (Paris) 44-C3, 429 (1983).

[3] J. P. Sethna and S. Kivelson Phys. Rev. B 26, 3513 (1982).

[4] A. J. Heeger, S. Kivelson, J. R. Schrieffer and W. -P. Su, Rev. Mod. Phys. 60, 781 (1988).

[5] A. S. Sidddiqui, J. Phys. C 13, 2147 (1980).

[6] S. Möller, G. Weiser and C. Lapersonne-Mayer, Syn. Met. 116, 23 (2001).

[7] S. A. Brazovskii and N. N. Kirova, JETP Lett. 33, 4 (1981).

[8] D. K. Campbell and A. R. Bishop, Phys. Rev. B 24, 4859 1981.

[9] Y. Onodera, Phys. Rev. B 30, 775 (1984).

[10] J. L. Bredas et al., J. Chem. Phys. 75, 255 (1981).

[11] K. Kuriyama, H. Kikuchi and T. Kajiyama, Langmuir 14, 1180 (1998).

[12] S. Kivelson and J. P. Sethna, J. de Physique (Paris), 44C3, 657 (1983).

[13] B. Horovitz, A. R. Bishop. and S. R. Phillpot, Phys. Rev. A 40, 1240 (1989).

[14] W. Franz, Z. Naturforsch. 13, 484 (1958); L. V. Keldysh, Sov. Phys. JETP 7, 788 (1958). 